\title{
The pathways and mechanisms of muramyl dipeptide transcellular transport mediated by PepT1 in enterogenous infection
}

\author{
Guo-Guang Ma ${ }^{1 \#}$, Bin Shi ${ }^{2 \#}$, Xue-Peng Zhang ${ }^{1}$, Yue Qiu ${ }^{1}$, Guo-Wei Tu ${ }^{1}$, Zhe Luo ${ }^{1,3}$ \\ ${ }^{1}$ Department of Critical Care Medicine, Zhongshan Hospital, Fudan University, Shanghai 200032, China; ${ }^{2}$ Department of Emergency Intensive Care \\ Unit, Yangpu Hospital, Tongji University, Shanghai 200090, China; ${ }^{3}$ Department of Critical Care Medicine, Xiamen Branch, Zhongshan Hospital, \\ Fudan University, Xiamen 361015, China \\ Contributions: (I) Conception and design: GW Tu, Z Luo; (II) Administrative support: GW Tu, Z Luo; (III) Provision of study materials or patients: \\ GG Ma, B Shi, XP Zhang; (IV) Collection and assembly of data: GG Ma, B Shi; (V) Data analysis and interpretation: GG Ma, B Shi, Y Qiu; (VI) \\ Manuscript writing: All authors; (VII) Final approval of manuscript: All authors. \\ \#These authors contributed equally to this work as co-first authors. \\ Correspondence to: Guo-Wei Tu; Zhe Luo. Department of Critical Care Medicine, Zhongshan Hospital, Fudan University, No. 180 Fenglin Road, \\ Xuhui District, Shanghai 200032, China. Email: tu.guowei@zs-hospital.sh.cn; luo.zhe@zs-hospital.sh.cn.
}

\begin{abstract}
Background: The transcellular transport of muramyl dipeptide (MDP) mediated by peptide transporter (PepT1) involves the translocation into intestinal epithelial cell (IEC) stage and the transport out of IEC stage. However, its mechanism has not been fully understood. This study aimed to investigate the pathways and mechanisms of MDP transcellular transport in enterogenous infection.

Methods: Firstly, experimental rats were randomly divided into three groups: sham-operation (sham group), MDP perfusion (MDP group), and PepT1 competitive inhibition (MDP + Gly-Gly group). Then, the overall survival (OS) and intestinal weight were measured in MDP and MDP + Gly-Gly group. HE staining was performed to observe the pathological changes of the small intestine. The levels of IL-6, IL-1b, IL-8, IL-10, TNF- $\alpha$, and nitric oxide (NO) in rat serum and small intestine were determined by ELISA. To further verify the pathways and mechanisms of MDP transcellular transport from IEC in intestinal inflammatory damage, the NFкB inhibitor, PDTC, was used to treated lamina propria macrophages in small intestinal mucosa in sham, MDP, and MDP + Gly-Gly groups. Finally, the expression of CD80/86 and the antigen presentation of dendritic cells (DCs) were measured by flow cytometry.
\end{abstract}

Results: MDP infusion was able to induce death, weight loss, and intestinal pathological injury in rats. Competitive binding of Gly-Gly to PepT1 effectively inhibited these effects induced by MDP. As well, competitive of PepT1 by Gly-Gly inhibited inflammation-related cytokines induced by MDP in rat serum and small intestine. Furthermore, we also found that MDP transported by PepT1 contributes to activation of macrophages and antigen presentation of DCs.

Conclusions: PepT1-NFкB signal is pivotal for activation of intestinal inflammatory response and MDP transcellular transport.

Keywords: Muramyl dipeptide (MDP); PepT1; enterogenous infection; immune cells

Submitted Feb 22, 2019. Accepted for publication Jul 26, 2019.

doi: 10.21037/atm.2019.07.103

View this article at: http://dx.doi.org/10.21037/atm.2019.07.103

\section{Introduction}

Intestinal infection is one of the leading causes of death in patients with severe trauma/burns, major surgery, hemorrhagic shock, and severe acute pancreatitis (1).
Under the above pathological conditions, intestinal bacteria invades peripheral blood through the damaged intestinal mucosa and further leads to bacterial translocation, which can induce systemic inflammatory responses, sepsis, 
and multiple-organ dysfunction syndrome (MODS) $(2,3)$. The intestinal barriers mainly consist of intestinal epithelial barrier, intestinal microbial barrier, and mucosal immune barrier (4). The translocation of bacteria and its products through the intestinal barrier were as follows: (I) breakthrough of the tight junction of the paracellular pathway; (II) increase of intestinal mucosal permeability due to apoptosis of intestinal epithelial cells (IECs) $(5,6)$. Although there is a certain understanding of intestinal bacterial translocation, it is not fully clear how these bacteria pass through complex intestinal barriers to form bacterial translocation (7).

The bacterial products can be transported into the intestinal epithelium via epithelial cell peptide transporter (PepT1) (8). In vivo and in vitro studies have shown that PepT1 mediates the transport of $\mathrm{N}$-formylated inflammatory peptides, such as $\mathrm{N}$-formylmethionyl, leucyl, and phenylalanine, which finally induces inflammation in the intestine $(9,10)$. Also, the ulcerative colitis and Crohn's disease can induce the PepT1 expression in the colon (11). The mechanism may be that alteration of intestinal flora promotes the excessive secretion of inflammatory peptides, which induce the ectopic expression of PepT1 as a substrate of PepT1. N-Formyl-Met-Leu-Phe (fMLP) is a neutrophil chemotactic peptide that can induce leukocyte chemotaxis and macrophage activation (12). Higher levels of fMLP transported by PepT1 can lead to large number of neutrophil aggregation, resulting in intestinal mucosal damage (13). However, fMLP is mainly a product of Escherichia coli, and it is not clear whether other bacterial products will cause intestinal damage through PepT1.

Muramyl dipeptide (MDP) is the decomposition product of Gram-negative and Gram-positive bacterial peptidoglycan, which is released during the growth and division of bacteria (14). Our previous study has confirmed that MDP-induced intestinal injury begins with the transcellular pathway mediated by PepT1 (15). MDP entering the IECs results in inflammatory damage through its effect on the nucleotide-binding oligomerization domain (NOD) and further activates inflammatory amplification signals $(16,17)$. After entering the IECs mediated by PepT1, the bacterial products can be transported out of the IECs through the velocity limiting transporter of the basement membrane, which may lead to systemic inflammatory responses (18). But, the subsequent action of MDP after being transported out of IECs still remains unclear. We speculated that it may activate mucosal lamina propria immune cells or enter peripheral blood circulation. Chamaillard et al. reported that PepT1 could be expressed on the surface of mammalian immune cells (19). After exposure to bacterial product, MDP, the expression level of NOD2 mRNA was significantly increased in neutrophils, macrophages, and peripheral blood mononuclear cells, and further promoted the expression of major histocompatibility complex 1 (MHC1) in macrophages (20). In short, these results not only indicate that MDP may activate immune responses through PepT1/NOD pathway, but also suggest that bacterial products have the potential to cause inflammatory amplification and systemic inflammatory responses after transcellular translocation. As a result of these findings, in this study, we attempted to explore the effects and mechanisms of MDP translocation in small intestinal cell, and further revealed the pathways of MDP translocation out of IECs.

\section{Methods}

\section{Experimental animals}

Eighty female Sprague Dawley rats weighing 250-300 g were purchased from Shanghai Experimental Animal Center of Chinese Academy of Sciences (Shanghai, China) and were housed under SPF conditions. Rats were provided with adequate food and water, fasting for 1 day before the experiment. The animals were treated in accordance with the Institute of Laboratory Animal Science standards concerning the care and use of laboratory animals, and the study protocol was approved by Animal Ethical Committee of Zhongshan Hospital, Fudan University (2018-046). During the experiment, the rats were randomly divided into three groups: sham-operation (sham group), MDP infusion (MDP group), and PepT1 competitive inhibition (MDP-Gly-Gly group), and the detail was described by our previous study (15). The primary outcome was overall survival (OS) and intestinal weight.

\section{Hematoxylin and eosin (HE) staining}

The small intestine tissues $(1-2 \mathrm{~cm})$ were fixed in $4 \%$ paraformaldehyde for $24 \mathrm{~h}$ and embedded in paraffin. Then, $5 \mu \mathrm{m}$ thickness of paraffin sections were stained with $\mathrm{HE}$ (Beyotime, C0105). Five high-power fields (HPF)/section were randomly selected to observe the pathological changes of the small intestine in each group. 


\section{Enzyme-linked immunosorbent assay (ELISA)}

Blood samples were collected from the rats. Then, the blood samples were centrifuged for $15 \mathrm{~min}(2,000 \mathrm{rpm})$. Serum levels of IL-6, IL-1b, IL-8, IL-10, TNF- $\alpha$, and nitric oxide (NO) were measured by ELISA (DAKEWE) according to the manufacturer's instructions.

The small intestinal mucosa was scraped and homogenized at $4{ }^{\circ} \mathrm{C}$ for $30 \mathrm{~min}$. The expression levels of IL-6, IL- $1 \mathrm{~b}$, IL$8, \mathrm{IL}-10, \mathrm{TNF}-\alpha$, and NO in rat small intestinal mucosa were measured by ELISA (DAKEWE) according to the manufacturer's instructions.

\section{Isolation of lamina propria macrophages}

Immersing small intestinal mucosa in $75 \%$ ethanol. Then, immersing them with PBS for $5 \mathrm{~min}$ and leaving them in Dulbecco's Modified Eagle Medium (DMEM, $4{ }^{\circ} \mathrm{C}$ ). The samples were centrifuged for $5 \mathrm{~min}(500 \mathrm{rpm})$ and were suspended with complete medium. Next, adding macrophage colony-stimulating factor (MCSF) $(20 \mathrm{ng} / \mathrm{mL}$, Jianglai Biological Technology) and changing medium with MCSF every 3 days. The mucosal lamina propria macrophages were ready for the following experiment at the 6th day. The NFאB inhibitor, PDTC, was purchased from Beyotime (S1809).

\section{Flow cytometry}

The expression levels of CD80/86 on the surface of dendritic cells (DCs) and the antigen presentation of DCs were measured by flow cytometry (CytoFLEX LX, BECKMAN).

\section{Statistical analysis}

All statistical analyses were carried out using IBM SPSS Statistics 21. The differences between each group were analyzed by one-way analysis of variance (ANOVA). It was considered to be statistically significant when $\mathrm{P}<0.05$.

\section{Results}

\section{PepT1 mediated MDP transport induces small intestinal pathological injury}

First, we found that MDP infusion was able to timedependently induce death and intestinal weight loss of rats. Then, according to the characteristics of transport peptides of PepT1, we used dipeptide Gly-Gly to competitively inhibit the transport of MDP. The results showed that GlyGly had significant protective effects on MDP-induced death and intestinal weight loss of rats, indicating that MDP transport is mediated by PepT1 (Figure 1A,B). By histologic analysis, we found that the small intestine in MDP group was characterized by structural disorganization and numerous inflammatory cell infiltration, exudation, and bleeding. But, compared with the MDP group, the histopathological changes in the group MDP + Gly-Gly (competition inhibition of PepT1 group) were significantly alleviated (Figure 1C). The histological results proved that MDP-induced structural damages of intestine were through PepT1 mediated transport, which validated the mechanism of MDP toxicity in rat model, and we carried out the following experiments on the verified model.

\section{Expression levels of serum $I L-6, I L-1 b, I L-8, I L-10$, TNF- $\alpha$, and NO}

We detected the inflammation-related factors in serum of the rats. As shown in Figure 2, compared to the sham group, the expression levels of IL-6, IL-1b, IL-8, IL-10, TNF- $\alpha$, and $\mathrm{NO}$ in rat serum were significantly higher in MDP group. However, competitive binding of Gly-Gly to PepT1 effectively inhibited these inflammation-related cytokines induced by MDP. The levels of IL-6, IL- 8 and TNF- $\alpha$ were as quality controls of the detection, of which the trends were in line with the published data. Moreover, the expression level of IL-6 had the largest difference in MDP + Gly-Gly group compare with the MDP group, while the anti-inflammatory cytokine IL-10 had a lower difference.

\section{Expression levels of $I L-6, I L-1 b, I L-8, I L-10, T N F-\alpha$, and NO in rat small intestinal mucosa}

As shown in Figure 3, MDP could induce higher levels of inflammatory cytokines IL-6, IL-1b, IL-8, IL-10, TNF- $\alpha$, and $\mathrm{NO}$ in rat small intestinal mucosa compared to the sham-operated group. But, the competitive inhibition of PepT1 by Gly-Gly significantly reduced the expression levels of IL-6, IL-1b, IL-8, IL-10, TNF- $\alpha$, and NO induced by MDP in rat small intestine. These suppressive effects were generally weaker than those in serum, and the cytokine levels in rat small intestine were also lower than those in rat serum. In addition, competitive binding of Gly-Gly to PepT1 even had a promoting effect on anti-inflammatory cytokine IL-10. The overall results are consistent with the results in Figure 2. 

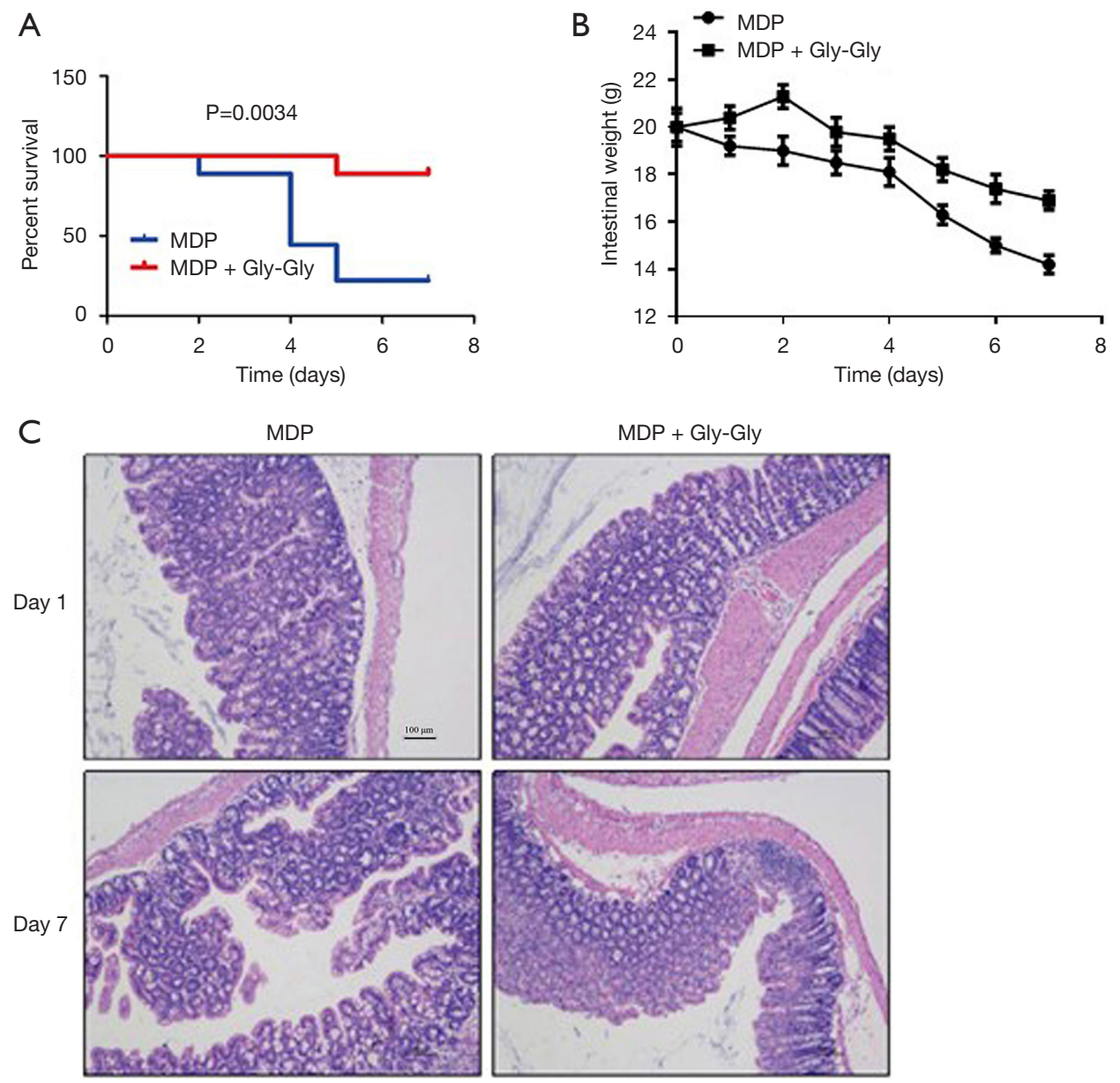

Figure 1 PepT1 mediated MDP transport induces small intestinal pathological injury. (A) The survival in each group was assessed in MDP and MDP + Gly-Gly groups. (B) The intestinal weight was measured at the 1st, 2nd, 3rd, 4th, 5th, 6th, and 7th day in MDP and MDP + Gly-Gly groups. (C) HE staining of small intestine in each group. 100× magnification. PepT1, peptide transporter; MDP, muramyl dipeptide; HE, hematoxylin and eosin.

\section{MDP transported by PepT1 contributes to activation of macrophages and antigen presentation of $D C s$}

Next, in order to confirm the relationship between MDP and immune cells, ELISA was performed to determine the expression levels of related immune molecules on the surface of macrophages isolated from mice. As shown in Figure 4A, IL-6, TNF- $\alpha$, and NO were highly expressed after MDP infusion in macrophages. Then, we found that Gly-Gly could inhibit MDP-induced activation of IL-6, TNF- $\alpha$, and NO in macrophages, suggesting that MDP transcellular translocation out of IECs was mediated by PepT1, and it was able to activate immune cells. Notably, the results also indicated that the effect of Gly-Gly competitive inhibition of PepT1 on macrophage activation and cytokine secretion induced by MDP could be simulated by $\mathrm{NF \kappa B}$ inhibitor PDTC. Furthermore, the results from flow cytometry indicated that MDP promoted the expression levels of CD80 and CD86 in DCs compared to the sham-operated group. But, the competitive inhibition of PepT1 by Gly-Gly significantly decreased the expression levels of CD80/86 induced by MDP and suppressed the antigen presenting ability in rat DCs (Figure 4B,C).

\section{Discussion}

When bacteria and their products invade or contact the body, the host cells can recognize the pathogen-associated 

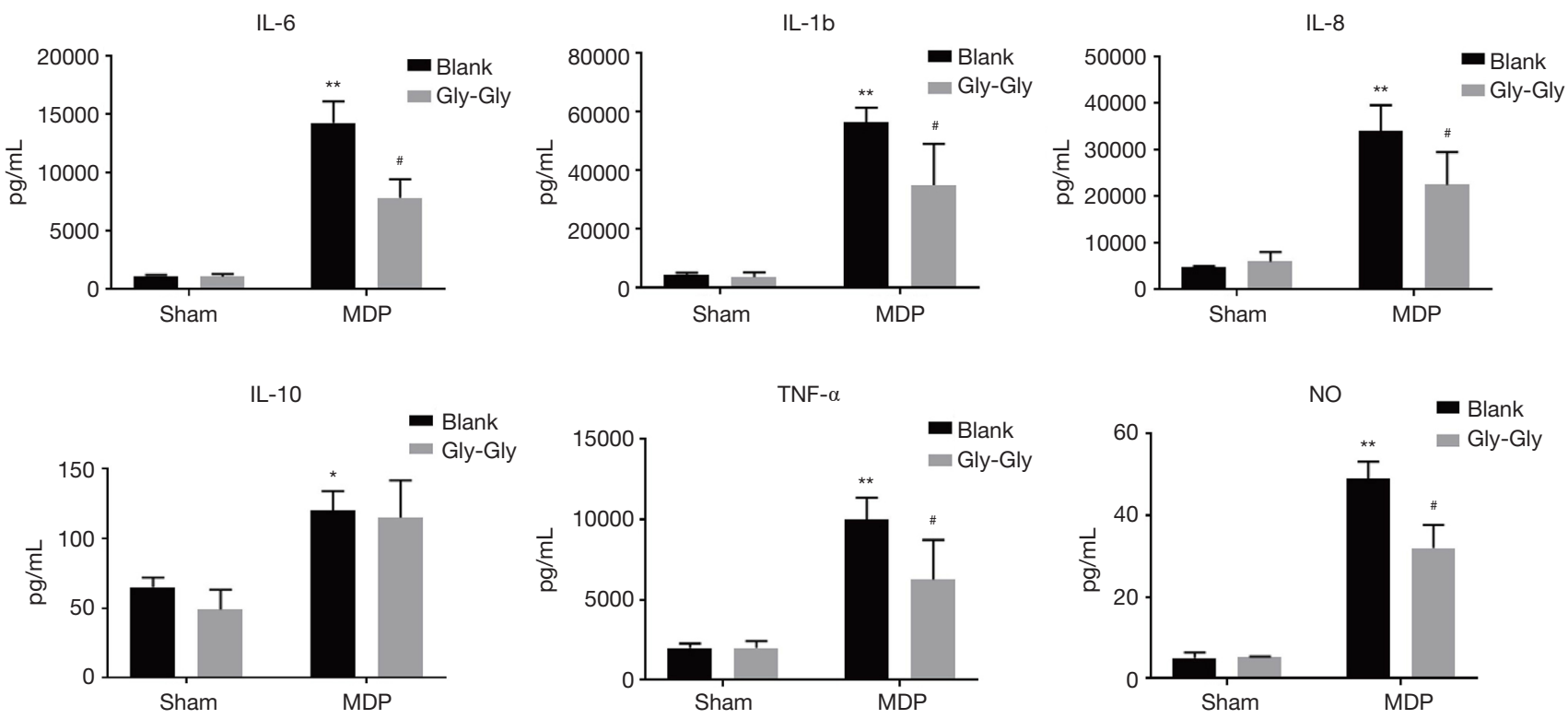

Figure 2 The expression levels of serum IL-6, IL-1b, IL-8, IL-10, TNF- $\alpha$, and NO were measured by ELISA. * $\mathrm{P}<0.05$, ** $\mathrm{P}<0.01$ versus sham group; ${ }^{\mathrm{P}}<0.05$ versus MDP group. NO, nitric oxide; ELISA, enzyme-linked immunosorbent assay; MDP, muramyl dipeptide.
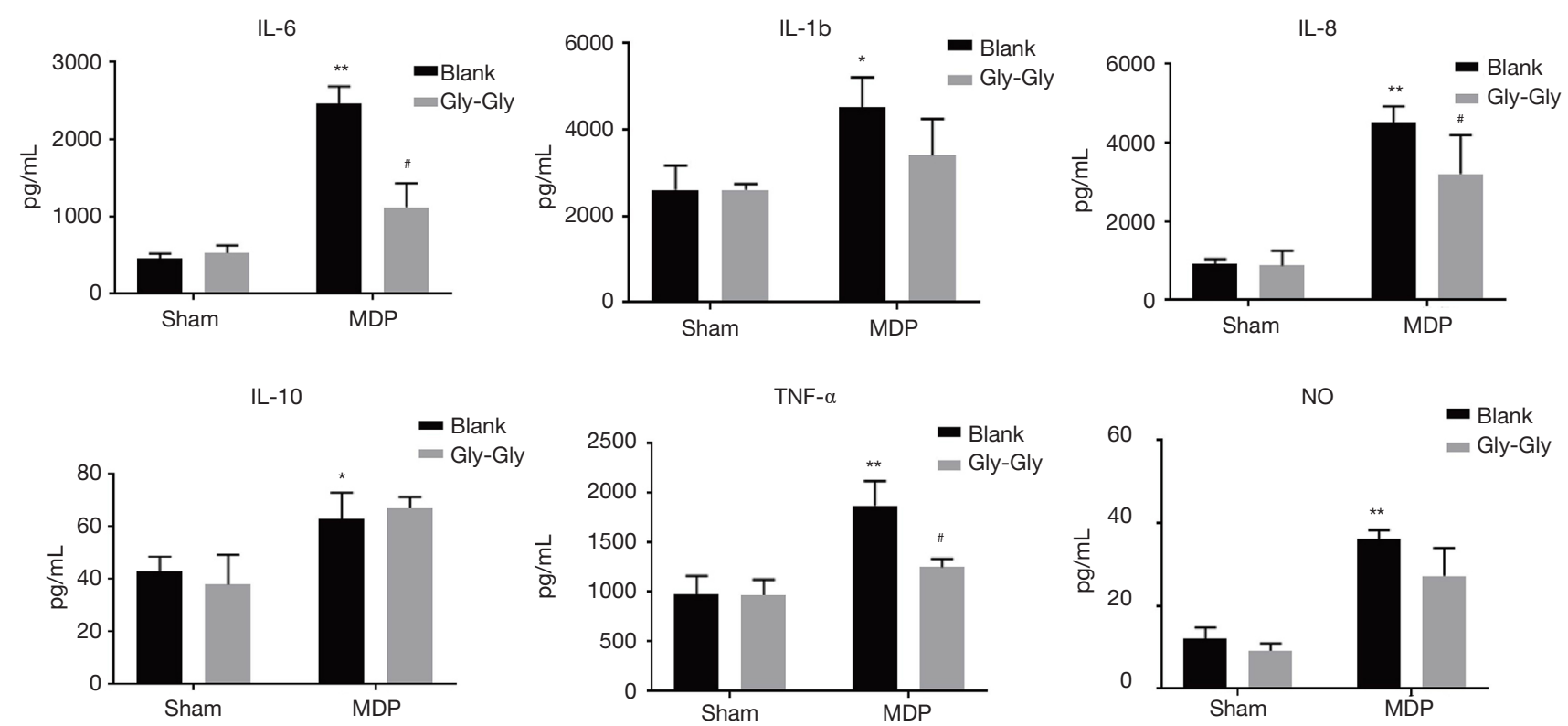

Figure 3 The expression levels of IL-6, IL-1b, IL-8, IL-10, TNF- $\alpha$, and NO in rat small intestinal mucosa were measured by ELISA. ${ }^{*} \mathrm{P}<0.05,{ }^{* *} \mathrm{P}<0.01$ versus sham group; ${ }^{\#} \mathrm{P}<0.05$ versus $\mathrm{MDP}$ group. NO, nitric oxide; ELISA, enzyme-linked immunosorbent assay; MDP, muramyl dipeptide. 

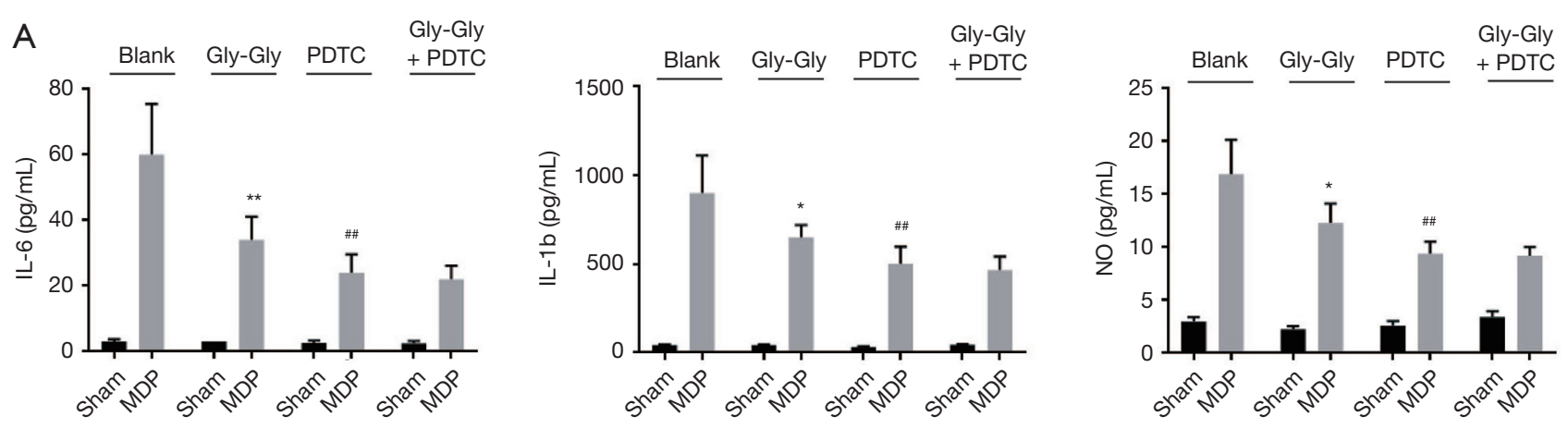

B
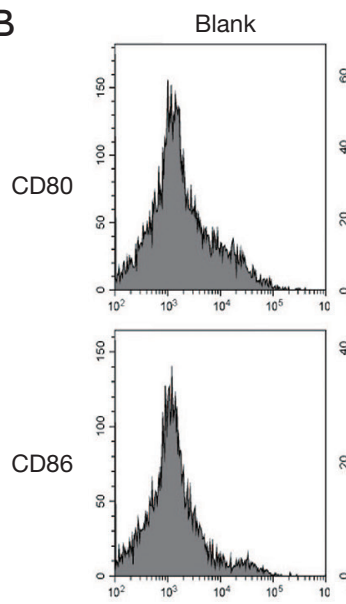

MDP
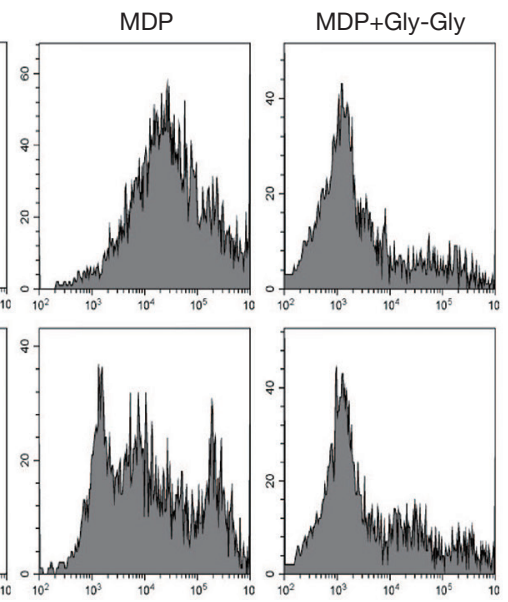

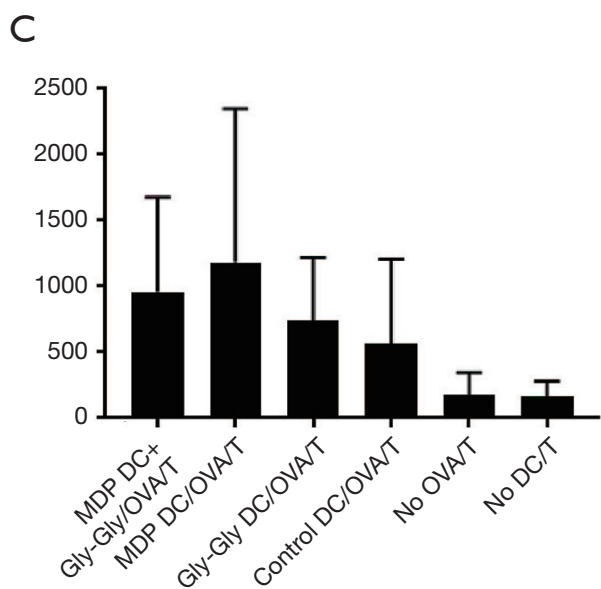

Figure 4 MDP transported by PepT1 contributes to activation of macrophages and antigen presentation of DCs. (A) The expression levels of serum IL-6, TNF- $\alpha$, and NO in rat macrophages were measured by ELISA. ${ }^{*} \mathrm{P}<0.05,{ }^{* *} \mathrm{P}<0.01$ versus MDP-Blank; ${ }^{\# \#} \mathrm{P}<0.01$ versus MDP-Blank. (B) The expression levels of CD80 and CD86 in rat DCs were measured by flow cytometry. (C) The antigen presentation of rat DCs were measured by flow cytometry. MDP, muramyl dipeptide; PepT1, peptide transporter; DC, dendritic cell; NO, nitric oxide.

molecular pattern (PAMP) through the pattern recognition receptor (PRR) (21). Activation of the downstream signals of PRR causes protective responses against infection, including releasing inflammatory cytokines, recruiting phagocytes, and DCs to mediate immune responses (22). The family of NOD proteins is an important intracellular PRR, and it is contained in IECs and immune cells. NOD1 and NOD2 are two representative receptor proteins in this family (23). MDP is a widespread bacterial product produced by all Gram-negative and positive bacteria. It is the smallest active peptidoglycan motif in cells and the ligand for NOD2 (24). MDP transported by PepT1 can induce the activation of $\mathrm{NF} \kappa \mathrm{B}$ by NOD2, which in turn initiates the expression of related genes involved in the innate immune response and the release of inflammatory factors. MDP also cause inflammatory bowel disease through a NOD2-dependent signal response (16).
The transcellular transport of bacterial products mediated by PepT1 is divided into two phases: (I) the translocation of bacterial products into IECs; and (II) the transport of bacterial products out of IECs $(25,26)$. In the present study, according to the characteristics of transport peptides of PepT1, Gly-Gly was used to competitively inhibit the functions of PepT1 after MDP infusion. The results indicated that the inflammatory injuries of small intestine induced by MDP were significantly eased after PepT1 was inhibited. As well, the competitive binding of Gly-Gly to PepT1 effectively decreased the expression levels of IL-6, IL-1b, IL-8, IL-10, and NO in rat serum and small intestine, indicating that MDP-induced small intestinal tissue injury and inflammatory response were mediated by PepT1.

Next, we continued to explore the pathways and mechanisms of PepT1-mediated transcellular translocation 
of MDP across IECs. Inflammation is a reaction against invading bacteria. The bacteria that invade the intestine are transported to the lymphoid follicles. Inflammation is a response to invading bacteria, which is transported to the lymphatic follicles, where macrophages and DCs have membrane receptors that bind to the surface components of the bacteria and further internalize the bacteria (27). This is the body's first line of defense against pathogens. These immune cells release cytokines to mobilize more phagocytes and effector molecules to the site of infection and induce inflammatory response (28). Inflammatory factors include IL-6, IL-1, IL- 8 and TNF- $\alpha$. IL-1 also plays a role in tissue repair, and IL-6 can induce the production of antiinflammatory proteins. The anti-inflammatory cytokines are IL-4 and IL-10. Macrophages are the main source of cytokines. Additionally, macrophages and neutrophils can initiate the synthesis of NO under cytokine stimulation (29). Yang et al. found that MDP has the functions of activating macrophages and promoting the production of IL-1, IL-2, and TNF- $\alpha$, non-specifically enhancing the body immune functions (30). The present study revealed that PepT1 was able to mediate the interaction of MDP and macrophages. Importantly, our data also indicated that the effect of GlyGly competitive inhibition of PepT1 on macrophage activation and cytokine secretion induced by MDP could be simulated by NFאB inhibitor PDTC. Collectively, these results suggest that macrophages can be activated during transcellular transport of MDP out of IECs. In addition, the PepT1-NFкB signaling pathway may play a pivotal role in activation of intestinal inflammatory responses and the transport of MDP out of IECs. DCs, as the most effective antigen presenting cells (APCs), involve in the initiation of adaptive immune response. After DCs are activated, the expression of MHC molecules on the cell surface is enhanced, and cytokines such as IL-1, IL-6 and IL-12 are secreted. As a result, the antigen presentation ability will be enhanced (31). Finally, our study found that MDP transported by PepT1 promoted the expression of membrane molecules CD80 and CD86 and enhanced antigen presentation of DCs.

In conclusion, our results indicated that PepT1-NFкB signal is pivotal for activation of intestinal inflammatory response and MDP transcellular transport.

\section{Acknowledgments}

Funding: This study was supported by grants from the Research Funds of Zhongshan Hospital (Grant No.:
2016ZSQN23, 2019ZSYXQN34, 2019ZSQN13 and 2018ZSQN53), and the Science Research Fund of Shanghai Municipal Health Commission (Grant No.: 2019ZB0105 and 201840314).

\section{Footnote}

Conflicts of Interest: The authors have no conflicts of interest to declare.

Ethical Statement: The study was approved by Animal Ethical Committee of the Zhongshan Hospital, Fudan University. The authors are accountable for all aspects of the work in ensuring that questions related to the accuracy or integrity of any part of the work are appropriately investigated and resolved.

\section{References}

1. MacDonald TT, Carter PB. Cell-mediated immunity to intestinal infection. Infect Immun 1980;28:516-23.

2. Roth RI, Owen RL, Keren DF, et al. Intestinal infection with Mycobacterium avium in acquired immune deficiency syndrome (AIDS). Dig Dis Sci 1985;30:497-504.

3. Macfarlane-Smith LR, Ahmed S, Wilcox MH. Molecular versus culture-based testing for gastrointestinal infection. Curr Opin Gastroenterol 2018;34:19-24.

4. Groschwitz KR, Hogan SP. Intestinal barrier function: Molecular regulation and disease pathogenesis. J Allergy Clin Immunol 2009;124:3-20.

5. Deitch EA. The Role of Intestinal Barrier Failure and Bacterial Translocation in the Development of Systemic Infection and Multiple Organ Failure. Arch Surg 1990;125:403-4.

6. Watson AJ, Hughes KR. TNF- $\alpha$-induced intestinal epithelial cell shedding: implications for intestinal barrier function. Ann N Y Acad Sci 2012;1258:1-8.

7. Balzan S, De AQC, De CR, et al. Bacterial translocation: overview of mechanisms and clinical impact. J Gastroenterol Hepatol 2007;22:464-71.

8. Buyse M, Berlioz F, Guilmeau S, et al. PepT1-mediated epithelial transport of dipeptides and cephalexin is enhanced by luminal leptin in the small intestine. J Clin Invest 2001;108:1483-94.

9. Ganapathy ME, Huang W, Wang H, et al. Valacyclovir: a substrate for the intestinal and renal peptide transporters PEPT1 and PEPT2. Biochem Biophys Res Commun 1998;246:470-5. 
10. Sugawara M, Huang W, Fei YJ, et al. Transport of valganciclovir, a ganciclovir prodrug, via peptide transporters PEPT1 and PEPT2. J Pharm Sci 2000;89:781-9.

11. Hu Y, Smith DE, Ma K, et al. Targeted Disruption of Peptide Transporter Pept1 Gene in Mice Significantly Reduces Dipeptide Absorption in Intestine. Mol Pharm 2008;5:1122-30.

12. Yasaka T, Boxer LA, Baehner RL. Monocyte aggregation and superoxide anion release in response to formylmethionyl-leucyl-phenylalanine (FMLP) and plateletactivating factor (PAF). J Immunol 1982;128:1939-44.

13. Buyse M, Tsocas A, Walker F, et al. PepT1-mediated fMLP transport induces intestinal inflammation in vivo. Am J Physiol Cell Physiol 2002;283:C1795-800.

14. Viswanathan VK. Muramyl dipeptide. Gut Microbes 2014;5:275-6.

15. Ma G, Shi B, Liu J, et al. Nod2-Rip2 Signaling Contributes to Intestinal Injury Induced by Muramyl Dipeptide Via Oligopeptide Transporter in Rats. Dig Dis Sci 2015;60:3264-70.

16. Yang Y, Yin C, Pandey A, et al. NOD2 Pathway Activation by MDP or Mycobacterium tuberculosis Infection Involves the Stable Polyubiquitination of Rip2. J Biol Chem 2007;282:36223-9.

17. Yang Z, Fuss IJ, Watanabe T, et al. NOD2 transgenic mice exhibit enhanced MDP-mediated down-regulation of TLR2 responses and resistance to colitis induction. Gastroenterology 2007;133:1510-21.

18. Dalmasso G, Nguyen HT, Ingersoll SA, et al. The PepT1NOD2 signaling pathway aggravates induced colitis in mice. Gastroenterology 2011;141:1334-45.

19. Chamaillard M, Girardin SE, Viala J, et al. Nods, Nalps and Naip: intracellular regulators of bacterial-induced inflammation. Cell Microbiol 2003;5:581-92.

20. Hedl M, Abraham C. Distinct roles for Nod2 and autocrine IL-1 $\beta$ in MDP-induced MAPK activation and cytokine secretion in human macrophages. J Biol Chem

Cite this article as: Ma GG, Shi B, Zhang XP, Qiu Y, Tu GW, Luo $Z$. The pathways and mechanisms of muramyl dipeptide transcellular transport mediated by PepT1 in enterogenous infection. Ann Transl Med 2019;7(18):473. doi: 10.21037/ atm.2019.07.103
2011;286:26440-9.

21. Godfrey D, Rathjen JP. Recognition and Response in Plant PAMP-Triggered Immunity 2012.

22. Leavy O. Innate immunity: New PAMP discovered. Nature Reviews Immunology 2015;15:402-3.

23. Strober W, Murray PJ, Kitani A, et al. Signalling pathways and molecular interactions of NOD1 and NOD2. Nat Rev Immunol 2006;6:9-20.

24. Girardin SE, Boneca IG, Viala J, et al. Nod2 is a general sensor of peptidoglycan through muramyl dipeptide (MDP) detection. J Biol Chem 2003;278:8869-72.

25. Qandeel HG, Duenes JA, Zheng Y, et al. Diurnal expression and function of peptide transporter 1 (PEPT1). J Surg Res 2009;156:123-8.

26. Ayyadurai S, Charania MA, Xiao B, et al. Colonic miRNA expression/secretion, regulated by intestinal epithelial PepT1, plays an important role in cell-to-cell communication during colitis. PLoS One 2014;9:e87614.

27. Permanyer M, Bošnjak B, Förster R. Dendritic cells, T cells and lymphatics: dialogues in migration and beyond. Curr Opin Immunol 2018;53:173-9.

28. Owen KL, Parker BS. Beyond the vicious cycle: The role of innate osteoimmunity, automimicry and tumor-inherent changes in dictating bone metastasis. Mol Immunol 2019;110:57-68.

29. Steptoe A, Hamer M, Chida Y. The effects of acute psychological stress on circulating inflammatory factors in humans: a review and meta-analysis. Brain Behav Immun 2007;21:901-12.

30. Yang S, Takahashi N, Yamashita T, et al. Muramyl dipeptide enhances osteoclast formation induced by lipopolysaccharide, IL-1 alpha, and TNF-alpha through nucleotide-binding oligomerization domain 2-mediated signaling in osteoblasts. J Immunol 2005;175:1956-64.

31. Chhabra A. Functional antigen presenting cells generated from human dendritic cells (DC) derived induced pluripotent stem cell lines. J Immun Ther Cancer 2015;3:P206. 